\title{
Article \\ The Effect of Optogenetically Activating Glia on Neuronal Function
}

\author{
Cecilia Pankau (), Shelby McCubbin and Robin L. Cooper *(D)
}

Citation: Pankau, C.; McCubbin, S.; Cooper, R.L. The Effect of Optogenetically Activating Glia on Neuronal Function. Neuroglia 2021, 2, 57-67. https://doi.org/10.3390/ neuroglia2010007

Academic Editor: James St John

Received: 20 July 2021

Accepted: 18 October 2021

Published: 22 October 2021

Publisher's Note: MDPI stays neutral with regard to jurisdictional claims in published maps and institutional affiliations.

Copyright: (c) 2021 by the authors. Licensee MDPI, Basel, Switzerland. This article is an open access article distributed under the terms and conditions of the Creative Commons Attribution (CC BY) license (https:// creativecommons.org/licenses/by/ $4.0 /)$.
Department of Biology, University of Kentucky, Lexington, KY 40506-0025, USA; Cecilia.Pankau@uky.edu (C.P.); Shelby.McCubbin@uky.edu (S.M.)

* Correspondence: RLCOOP1@uky.edu

\begin{abstract}
Glia, or glial cells, are considered a vital component of the nervous system, serving as an electrical insulator and a protective barrier from the interstitial (extracellular) media. Certain glial cells (i.e., astrocytes, microglia, and oligodendrocytes) within the CNS have been shown to directly affect neural functions, but these properties are challenging to study due to the difficulty involved with selectively-activating specific glia. To overcome this hurdle, we selectively expressed light-sensitive ion channels (i.e., channel rhodopsin, ChR2-XXL) in glia of larvae and adult Drosophila melanogaster. Upon activation of $\mathrm{ChR} 2$, both adults and larvae showed a rapid contracture of body wall muscles with the animal remaining in contracture even after the light was turned off. During ChR2-XXL activation, electrophysiological recordings of evoked excitatory junction potentials within body wall muscles of the larvae confirmed a train of motor nerve activity. Additionally, when segmental nerves were transected from the CNS and exposed to light, there were no noted differences in quantal or evoked responses. This suggests that there is not enough expression of ChR2-XXL to influence the segmental axons to detect in our paradigm. Activation of the glia within the CNS is sufficient to excite the motor neurons.
\end{abstract}

Keywords: channel rhodopsin; Drosophila; glia; behavior

\section{Introduction}

From insects to mammals, the general role of glia in neural function is to serve as an electrical insulator and a protective barrier from the interstitial (extracellular) media or hemolymph (i.e., blood in insects and crustaceans). In mammals, the glial cells (i.e., astrocytes, microglia and oligodendrocytes) within the CNS are involved with many functions including the release of gliotransmitters and cytokines, the developmental and maintenance cues for neurons and their synapses and the maintenance of ionic homeostasis within their environment (see recent review by Sancho et al. [1]). Schwann cells have unique properties within the CNS. The Schwann cells are known to interact with axons bidirectionally to provide cues for the localization of ion channels and associated proteins, such as at the edges of internodes and within the nodes of Ranvier [2]. Considering that the mitochondrial types and densities vary within myelinated axons in the paranodes and nodes, it appears that this is related to homeostasis with ATP dependent pumps [2]. These regional differences then impact the local ionic flux at nodes and internodes differentially over the Schwann cell. The physiological function of glial cells being influenced by neural activity was demonstrated in the leech ganglion as $\mathrm{K}^{+}$efflux from the neurons' depolarized glia [3]. This is now assumed to be one of the major functions for oligodendrocytes and Schwann cells. Schwann cells at regions, such as at the neuromuscular junctions (NMJs), sense a specific ionic environment induced by the electrical activity in motoneuron terminals and the muscle [4,5]. ATP co-released from acetylcholine packaged synaptic vesicles at NMJs results in responses within the terminal Schwann cells [6,7]. Since there is such a dynamic interplay of the terminal Schwann cell, neuron and muscle, the NMJ is now referred to as a tripartite synapse [8,9]. 
There are multiple disease states related to glia dysfunction [10-12]. Generally, it has been challenging to selectively regulate the activity of glial cells around axons or in the CNS in order to examine the impact on the activity of neurons [13]. However, with the advent of the genetic modification of selective cells, one can regulate the activity of glia while also examining the function of neurons and the impact on synaptic transmission. Even using brain slices or neuronal cultures it is difficult to ascertain direct actions of compounds on neurons without the potential of influencing microglia or cells co-cultured, which can then impact neuronal function [14]. It is of interest to address the direct effects of the excitability of glia on neurons or on the glial cells themselves. In this study, we made use of optogenetics [15-17] to selectively excite glia while assessing neuronal activity and behavior in larval and adult $D$. melanogaster. In brain slices of mice, it was shown that glia stimulation, by the activation of channel rhodopsin, resulted in the release of glutamate, which then activated neurons and altered activity of the neural circuit [18]. There is hope in the future to be able to implement such procedures in the intact mammalian CNS to modulate disorders related to glial dysfunction.

D. melanogaster serve as a model for addressing many disease states among humans $[13,19-22]$ and have the potential to be used while assessing the potential use of new technology, such as optogenetics, in treating neuronal disfunction [15,21,22]. The impact of glia on neuronal development and function is also now being addressed in D. melanogaster models $[13,23]$. The glial cells in the CNS and segmental nerves of larval Drosophila have been described [13,23]. As mentioned by Stork et al. [23], only about $10 \%$ of cells within the CNS of Drosophila are glial cells, which potentially makes it easier to address their function. Of the types of glial cells, Stork et al. [23] described them as perineurial and subperineurial glial cells. These types of glia are within the axonal fascicles. What is described as 'wrapping glia' does not wrap around each axon individually, as a Schwann cell does in mammals, but wraps around a group of axons in a fascicle [23]. These glial cells are identified in electron micrographs of segmental nerve cross-sections [23]. There are also variations in the naming of the glia in Drosophila. MacNamee et al. [24] reviewed the current nomenclature as 'interface glia' [25]), 'longitudinal glia' [26], 'astrocyte-like glia' [27] and 'astrocytes' [23,28,29]. MacNamee et al. [25] wrote that the glial identities are based on their morphology and molecular characteristics; however, the glial cells described in mammals (i.e., astrocytes, microglia, oligodendrocytes or Schwann cell) are not exactly the same in morphology and there are fewer of them for larval Drosophila compared to mammals. They likely serve much of the same functions in mammals and Drosophila. The glia also form a barrier to large molecules from the hemolymph environment of the larvae and adult similar to the blood brain barrier in mammals [23]. MacNamee et al. [24] and Ng et al. [30] reported on these various types of glia in the segmental nerves and CNS, which can now be selectively identified by differences in molecular markers. In this study, a pan-glial driver was used to express the light sensitive ion channel (i.e., channel rhodopsin-XXL) in all glia cells, which allows one to depolarize the cell membrane to excite glia. Thus, as a proof of concept, one can now address how the acute activation of glia in D. melanogaster can impact larval and adult flies and allow for the examination of behaviors and neural activity in order to assess the effects in intact animals.

\section{Results}

\subsection{Larval and Adult Behavior}

Adults of UAS-ChR2 and glia > Chr2 XXL were fed ATR for 2 days prior to being examined in a vial and switched to a vial without food in order to examine the effect of blue light. Light was focused on the base of the vial while lightly taping the vial in the table to bring the flies to the bottom so the light could be directed on to them. Adults that did not become paralyzed were left to fly out of the vial. About $50 \%$ of the adults did not respond to blue light due to the non-homozygous parental glia; line (w[1118]; $\left.\mathrm{P}\left\{\mathrm{w}\left[+\mathrm{m}^{*}\right]=\mathrm{GAL} 4\right\} \mathrm{repo} / \mathrm{TM} 3, \mathrm{Sb}\right)$. To illustrate the effect on the adults that were sensitive to blue light, a close up movie of two adults is shown (Movie S1). Note how quickly they 
respond to the light and fall to the bottom of the vial and remain on their on their backs. For the controls, UAS-ChR2XXL was exposed to $30 \mathrm{~s}$ of light with the same approach as glia $>$ Chr2 XXL adults. With the six different groups of adults in vials, each for glia $>\mathrm{Chr} 2$ XXL and UAS-ChR2XXL, no adults showed a freezing behavior or laying on their back when exposed to blue light for all six vials of the UAS-ChR2XXL. Two glia > Chr2 XXL were combined with six adults of UAS-ChR2XXL for direct comparisons (Movie S2). All flies of glia $>\mathrm{Chr} 2 \mathrm{XXL}$ in the six vials showed the freezing behavior within $30 \mathrm{~s}$ of blue light exposure ( $\mathrm{N}=6$ vials, 5 adults of each line, $p<0.05$ rank sum Wilcoxon test, non-parametric). To better observe a group of blue light sensitive adults of glia $>\mathrm{Chr} 2 \mathrm{XXL}$, responders were placed in a large Petri dish and first observed under IR light. The adults do not fly around much in the dark (using only IR light) without some mechanical disturbance. So, the dish was shaken and rotated by hand in IR and during and after blue light exposure (Movie S3). Here one can see that the adults quickly freeze, but that there is a lot of variation in the time of recovery. Some adults recover as soon as the light is turned off and others take $3 \mathrm{~min}$ to recover. To insure they are paralyzed, the glass dish was mechanical shaken and rotated to stimulate movement after the blue light, as only IR light was being used while the flies were being observed by a camera screen. It was observed that some of the adults, upon recovery from blue light exposure, underwent a series of rapid twitches before being able to move around normally. This behavior was not observed in all the light sensitive adults as shown in the following video (Movie S4). With an enlarged view of adults in a small container more adults can be closely observed. Having a dim white light background promoted the flies to move around on their own without having to mechanically shake the dish as for IR only exposed adults. It was apparent that a rapid grooming behavior was present when the flies were recovering, and overall movements were slow but rapidly picked up in speed over $5 \mathrm{~min}$ as seen after a $30 \mathrm{~s}$ blue light was turned off compared to 4 min later (Movie S5).

Responses of larvae to blue light were examined while the larvae were crawling on a $1 \%$ apple juice agar plate. The larvae of glia $>\mathrm{Chr} 2 \mathrm{XXL}$ rapidly contract with exposure to blue light (Movie S6) while the larvae of UAS-ChR2XXL do not (Movie S7) ( $\mathrm{N}=20$ larvae of each line were examined, $p<0.05$ rank sum Wilcoxon test, non-parametric).

\subsection{Activity of Motor Neurons with Intact CNS}

In dissected larvae of Glia $>\mathrm{Chr} 2 \mathrm{XXL}$, a recording with an intracellular electrode in muscle six showed that blue light exposure resulted in a delayed response of a barrage of motor neuron activity. This resulted in EJPs that facilitated and depolarized the muscle in order to produce contraction (Figure 1A1). Longer blue light exposure resulted in a more enhanced response (Figure 1A2). If the CNS was removed, no alteration in the spontaneous quantal events or evoked responses was noted (Figure 1B). However, no induced responses occurred in the parental line (UAS-Chr2XXL) fed ATR for the same time period (Figure 1C). 


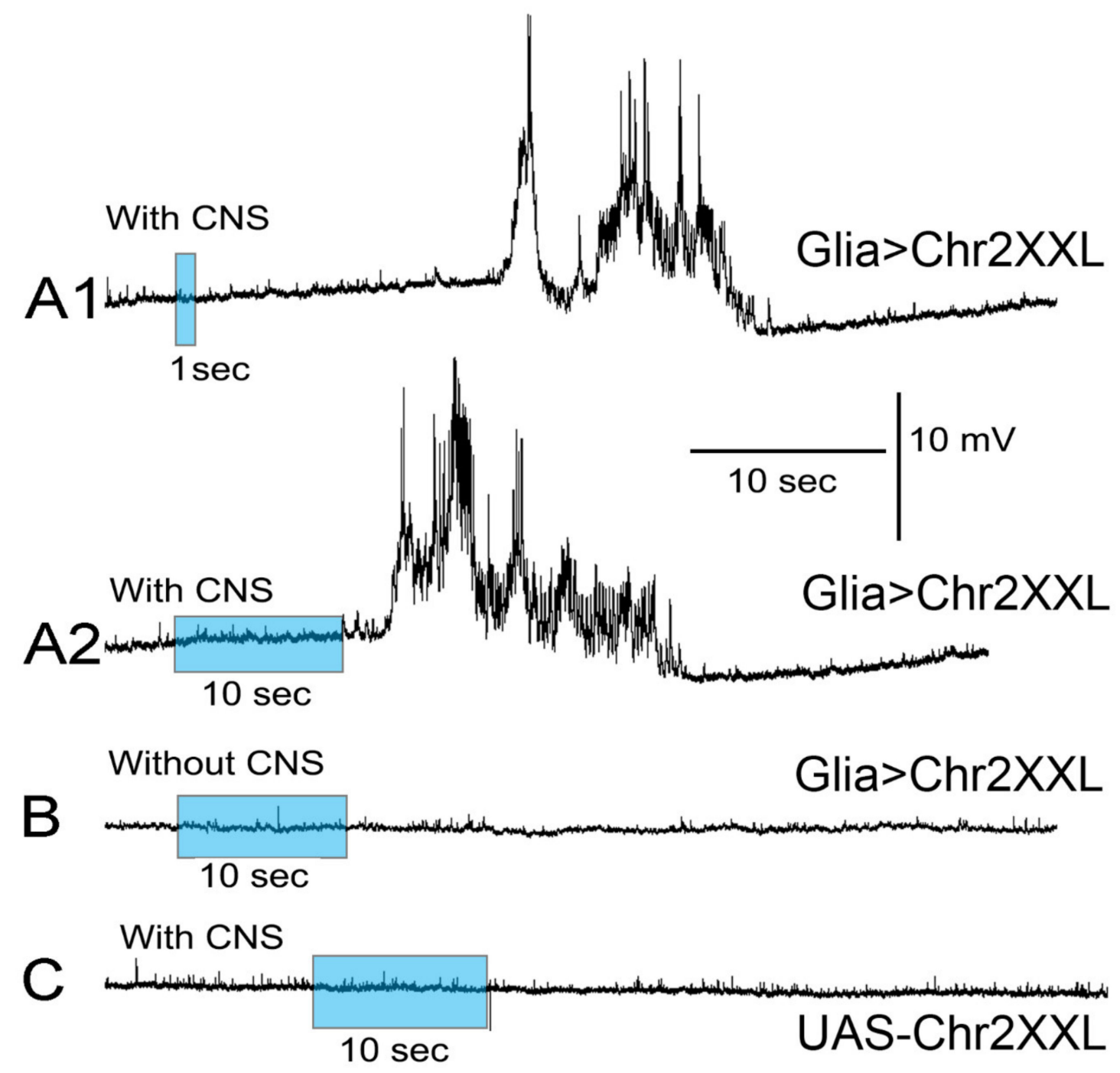

Figure 1. Responses measured in body wall muscles with intracellular recordings when glia within the CNS express channel rhodopsin are activated by blue light. (A1) Larvae (glia > Chr2 XXL) dissected and dark-adapted for 5 min while measuring membrane potential before and during a brief exposure to blue light and with a longer period (A2) of blue light exposure. The longer blue light exposure presented a more robust effect on membrane depolarization due to motor neuron activation. (B) If the CNS was removed, no alteration in the spontaneous quantal events or evoked responses occurred. (C) Larvae of the parental line (UAS-ChR2XXL) did not respond with motor neuron activation upon blue light exposure. All larvae were fed ATR for $24 \mathrm{~h}$ from the second instar stage and were maintained in the dark prior to viewing. The small deflections in the base line are quantal responses due to spontaneous vesicle fusion events. The large defections are excitatory junction potentials due to the motor neuron evoking synaptic transmission.

\subsection{Antibody Staining for Channel Rhodopsin}

The immunocytochemistry of the larval brain revealed an overall expression of the channel rhodopsin protein over the entire CNS with no highlighting of the segmental nerves branching from the CNS. The glial expressing line (Glia > Chr2XXL; Figure 2A) and a parental line (UAS-Chr2XXL; Figure 2B) are shown side by side. The images of the parent lines were dimmer with the same pinhole and laser intensity settings on the confocal microscope that the CNS could barely be made out while the Glia > Chr2XXL were robust with the intensity of the staining. The intensity of the CNS of the control was enhanced in Figure 2B to view the CNS. The segmental nerves did not reveal staining. 

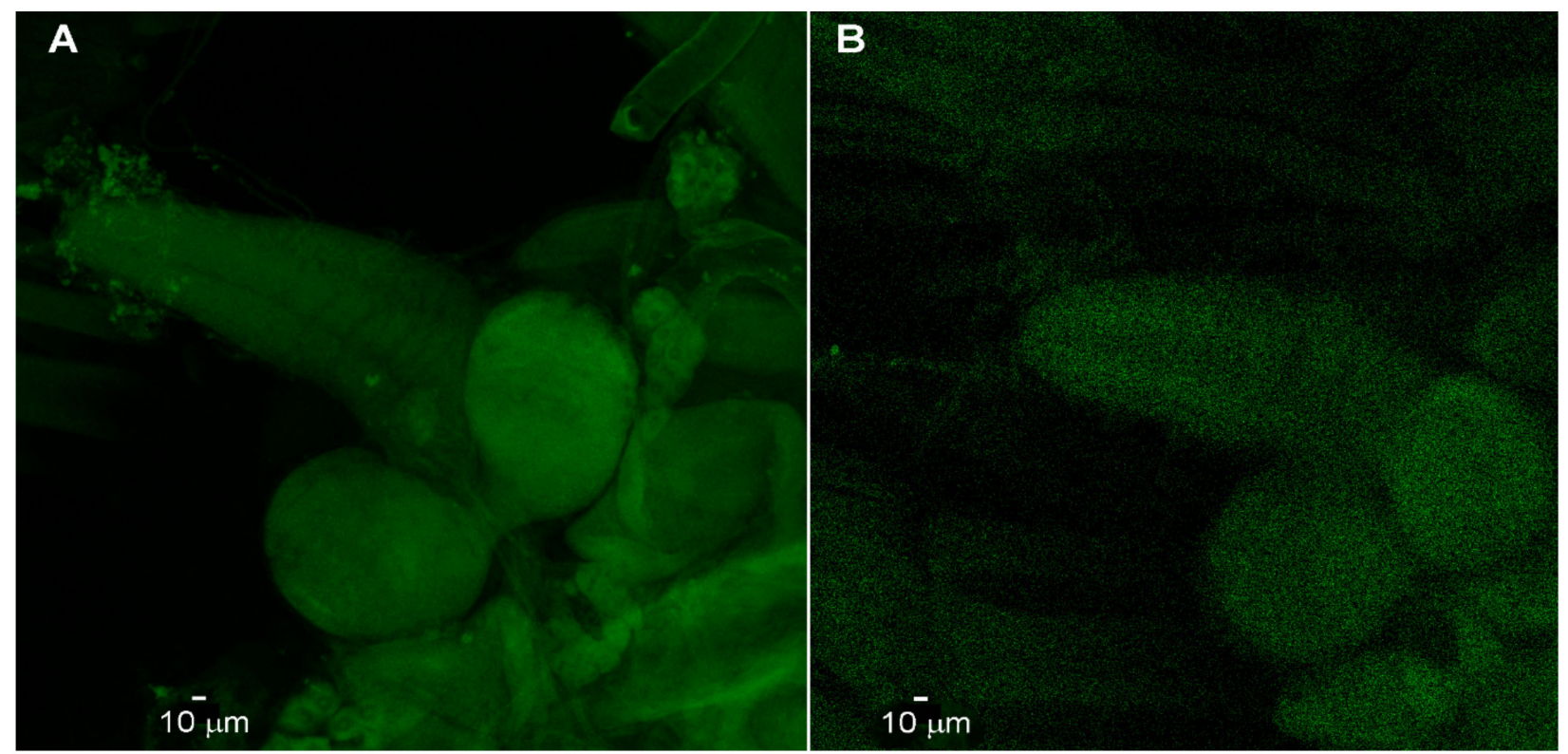

Figure 2. Immunocytochemistry for channel rhodopsin in the larval CNS. (A) Dissected preparation revealing the staining for anti-channel rhodopsin using a secondary FITC antibody for the larval CNS. (B) The parental line (UAS-ChR2XXL) did not reveal expression of channel rhodopsin using parallel processing of the tissue. The image in (B) is enhanced to observe the CNS due to the low intensity of the staining. When using the same intensity as in (A), the CNS is not visible for the controls. The segmental nerves in either line did not reveal any staining pattern for channel rhodopsin.

\section{Discussion}

Glial activation of intact larvae via channel rhodopsin activation results in the depolarization of the motor neurons and produces muscle contraction and paralysis, which was documented in direct recordings of muscle fibers in dissected larvae. The paralysis observed in the adults when stimulating glia in the same manner appears to also be due to muscle contraction as the legs rapidly shook prior to becoming immobile.

These studies indicate, as a proof of concept, that light-activated channels expressed in glia can induce enough motor neuron activity to produce what appears to be similar to an epileptic seizure in mammals and can result in temporary paralysis. Upon removal of the light stimulus, there was a short delay before the larvae or the adults could regain motor function and respond to environmental stimuli. Since all the glia within the CNS we re activated, the glial actions are likely to affect the entire neural circuitry of the sensory axon projections, interneurons and motor neurons. Given that Stork et al. [23] had shown septate junctions, via electron microscope imagining, among glia in the subperineurial layer in both the CNS and the PNS, the glia likely function as a syncytium. This may also account for the seizure-like behavior in the adults and larvae. This models the astroglial syncytium in epileptic seizure in mammals [23]. In electrophysiological measures of astrocytes in the CNS of third instar larvae, MacNamee et al. [24] did not find voltage-gated channels on the soma but could not rule out their existence in the finer processes. There appears to be a glutamate transporter that exchanges ions on the soma of astrocytes and the ion exchange can account for some of the ionic currents in astrocytes when the nerves are actively releasing glutamate [24]. In the mammalian CNS, astrocytes can impact $\mathrm{Ca}^{2+}$ signaling within neighboring neurons but this is location specific in the CNS [23,31,32]. In the Drosophila CNS, the dysfunctional cellular regulation of $\mathrm{Ca}^{2+}$ through the SERCA within astrocytes had an impact on circadian rhythm and olfaction $[13,30,33]$. How the local influence of glia on neurons occurs remains to be addressed.

When the CNS is removed, leaving the segmental nerves, exposure of these segmental nerves to blue light for over $30 \mathrm{~s}$ did not induce the depolarization of motor neurons enough to produce action potentials. This suggests that either there is not a high expression 
of ChR2XXL in the glia around the distal regions of segmental nerves, or there's not enough response from segmental glia to influence the segmental axons to be detected using our paradigm.

We do not yet know which neuronal cell types are initially activated by exciting glial cells with Chr2-XXL. Sensory input, interneurons or motor neurons, or a combination of these neuronal types, could have resulted in the motor neuron activation which was monitored from recordings made in muscle fibers. We hope that demonstrating that the activation of the glia in the CNS of larval and adult Drosophila results in behavioral changes will initiate further studies into the function of the various subtypes of glia within the CNS. Since the subtypes of glia have differences in molecular expression profiles, perhaps they could be selectively targeted for expression of light sensitive channels $[24,30]$. Using selective expression in different glial types of the thermal sensitive protein (TrpA1) in astrocytes in adult Drosophila resulted in altered circadian locomotor function and even olfaction $[24,30,33]$. It would be of interest to also explore this approach in larvae.

The potential off target response to light is addressed by the parental controls not responding in the same manner as the Glia > Chr2XXL line, as larvae do have the ability to sense light. Larvae have a light sensing organ in their head region (i.e., Bolwig organ) that when activated can result in photoavoidance [34]. It is likely this organ is responsible for light detection to maintain that the first through early third instar is negative phototactic while the late third instar is positive phototactic. However, the controls and the glia expressing channel rhodopsin showed drastically different responses to blue light exposure. Even with bright light activation of the Bolwig organ in wild type larvae they do not freeze and do not go into a state of paralysis during the light exposure [34]. One does need to be aware that there are light sensitive neurons on the body of larvae that are important when conducting optogenetic studies when various wavelengths of light are used [34].

Since the glia in the CNS is spread out over the CNS, as compared to the wrapping of segmental nerves, the large surface area of glia in the CNS may contain more expressedchannel rhodopsin. In addition, given the exposure area to the blue light it may have a greater effect on the glia in the CNS than for the glia around segmental nerves. If the glia releases $\mathrm{K}^{+}$ions when stimulated, resulting in the depolarization of the motor neurons, it is possible that the threshold is different in the axons as compared to the cell bodies and site of spike initiation within the regions of the motor neurons around the glia within the CNS than the axons of segmental nerves [35]. The high intensity LED blue lights can cause organelle damage [36] and potentially cause cell excitability; however, control lines are also exposed to the blue light and are also fed ATR to control for such off-target effects.

The anti-channel rhodopsin staining indicated a global expression in the CNS of the Glia > Chr2XXL line as compared to the parental line (UAS-ChR2XXL). In addition, the fluorescence was not observed in the segmental nerves of the Glia > Chr2XXL line with confocal imaging. This could be due to the primary and/or secondary antibody not having sufficient access to the target. Future studies with longer incubation times and trials in timing of Triton- $X$ exposure for permeabilization of the tissue for the antibodies to access the target antigens may resolve this issue.

We are now undertaking studies to determine the mechanisms of how glial activation alters neuronal function. It is likely that potassium ions are leaving the glial cells and depolarizing the local neurons, resulting in overall activation. Inhibitory circuits on motor function, as well as excitatory circuits, are likely influences, with the net result of the activation of the motor neurons. Future studies are needed to determine the cellular response from the activated glia, and potentially inactivated glia, in how specifically the neural circuitry is influenced in larvae as opposed to adults [24,30,33]. As a model, Drosophila offers some insight into disorders in glial function in mammals [13] and with more targeted future experiments an enhanced understanding of glia in Drosophila and mammals should be forthcoming. 


\section{Methods and Materials}

\subsection{Fly Stock Maintenance}

Flies utilized were obtained from Bloomington Drosophila Stock Center and were maintained in a laboratory. Third instar larvae were used for all electrophysiological studies. Flies and larvae were fed a standard cornmeal-agar-dextrose-yeast medium diet (modified from Lewis, [37]), housed at temperatures between 20 and $21^{\circ} \mathrm{C}$, and maintained on a 12:12 L:D cycle. The filial 1 (F1) generations were obtained by crossing females of y[1] $\mathrm{w}$ [1118]; PBac\{y[+mDint2] w[+mC] = UAS-ChR2.XXL\}VK00018 (BDSC stock \# 58374) with males using the pan-glial driver $\mathrm{w}[1118] ; \mathrm{P}\left\{\mathrm{w}\left[+\mathrm{m}^{*}\right]=\right.$ GAL4 $\}$ repo/TM3,Sb (BDSC stock \#7415). The F1 generation had 50\% sensitivity to blue light. They were screened prior to electrophysiology recordings to insure to we used larvae that were responsive to blue light.

A widely used approach in research using Drosophila is the UAS-Gal4 binary expression system, which can be used to target the expression of the effector gene of interest to a subset of cells in a controlled manner. The UAS-Gal4 binary expression system consists of two elements: yeast Gal4 transactivator and upstream activating sequence (UAS), which drives the expression of the reporter or effector gene [38]. In this study, we expressed channel rhodopsin in all glia cells. The parental lines (UAS-Chr2XXL) were also examined for the effect of light sensitivity for behaviors and electrophysiological studies.

\subsection{Light Stimulation}

All trans-Retinal (ATR; Sigma-Aldrich, St. Louis, MO, USA) was diluted in $50 \mathrm{~mL}$ of standard fly food to a final concentration of $200 \mu \mathrm{M}$ and protected from light with aluminum foil. All trans-Retinal is a cofactor for the channel rhodopsin, which increases its sensitivity to light and increases single channel conductance [15]. ATR (500 mg) was dissolved in $17.6 \mathrm{~mL}$ of absolute ethanol to make $100 \mathrm{mM}$ stock solutions. Stock solution was transferred to small tubes, wrapped with aluminum foil and kept in a $-20{ }^{\circ} \mathrm{C}$ freezer. The ATR should be kept away from light since it is sensitive to light. The ATR was either mixed well with the fly food or the fly food was dissolved in the microwave. The food was left to cool, then ATR or absolute ethanol was mixed with the food as a control. Both foods were stored in the refrigerator for 3 days with a cotton plug to allow the EtOH to evaporate. It had been noted that larval development slowed in the presence of ethanol, so precautions were taken to limit its developmental influence by evaporating the alcohol [39]. Only early third instar Drosophila larvae were used (50-60 h post hatching) and were fed ATR for $24 \mathrm{~h}$ before conducting electrophysiological or behavior assays.

The high-intensity LEDs used in this study were blue light (470 nm wavelength, LEDsupply, LXML-PB01-0040, $500 \mathrm{~mA}$ ) because literature determined that this wavelength would result in maximum activation of the light-sensitive proteins [40-43]. The photon flux (number of photons per second per unit area) was obtained with a LI-COR (model Li-1000 data Logger, LDL3774; LI-COR from Lincoln, NE, USA), which measured $\mu \mathrm{Mol} \mathrm{s}^{-1} \mathrm{~m}^{-2}$ per $\mu \mathrm{A}$. In addition, the full spectrum of the lights was measured with a Jazz (Ocean Optics Inc., Largo, FL, USA) to obtain a total $\mathrm{W} / \mathrm{m}^{2}$ from 340 to $800 \mathrm{~nm}$ spectrum for each light source. The ability to control the light stimulation was managed by Arduino system (2015 Arduino, LLC. https:/ / www.arduino.cc/ (accessed on 19 October 2021)).

\subsection{Electrophysiology}

The technique to dissect larvae is described $[19,43]$. In brief, a longitudinal dorsal midline cut was made in third instar larvae to expose the CNS. The preparation dish consisted of a glass slide with magnetic tape adhered to one side. A hole in the center of the magnetic strip allowed the preparation to be viewed from the bottom with transmitted light. This type of recording dish has been described previously for pinning ganglia isolated from the leech ventral nerve cord [44]. The segmental nerves were cut and sucked into a suction electrode, which was filled with saline and stimulated. The segmental nerves were stimulated at $0.5 \mathrm{~Hz}$ (S88 Stimulator, Astro-Med, Inc., Grass Co., West Warwick, RI, USA). To monitor the transmembrane potentials of the body wall muscle (m6) of third 
instar larvae, a sharp intracellular electrode (30 to $40 \mathrm{M} \Omega$ resistance) filled with $3 \mathrm{M} \mathrm{KCl}$ was used to impale the fiber. An Axoclamp 2B (Molecular Devices, Sunnyvale, CA, USA) amplifier and 1 X LU head stage were used. The excitatory junction potentials (EJPs) and spontaneous quantal responses (i.e., minis or mEJPs) were recorded online to a computer via a PowerLab/4sinterface (ADI Instruments, Colorado Springs, CO, USA). All events were measured and calibrated with the LabChart7 software (ADInstruments). Fly saline modified haemolymph-like 3 (HL3) was used: (in mmol/L) $70 \mathrm{NaCl}, 5 \mathrm{KCl}, 20 \mathrm{MgCl}_{2}$, $10 \mathrm{NaHCO}_{3}, 1 \mathrm{CaCl}_{2}, 5$ trehalose, 115 sucrose, $25 \mathrm{~N}$,N-bis(2-hydroxyethyl)-2-aminoethane sulfonic acid (BES) and pH at $7.1[43,45,46]$. Salts for the saline were obtained from SigmaAldrich Corporation (St. Louis, MO, USA).

\subsection{Larval and Adult Behavior}

Locomotive behavior was assessed by placing larvae on an apple juice $1 \%$ agar plate [47]. The larvae were left to acclimatize to their new environment for one minute. Then, locomotion activities were recorded with an IR camera, in which the camera provided the IR light source. The particular camcorder that was used (Panasonic $4 \mathrm{~K}$ video Camera Model HA-VX870 recorded at $72 \mathrm{Mb}$ per second for $3840 \times 2160$ resolution) was able to rapidly adjust to the light sensitivity from the LED to the IR lighting, which recorded activity when the blue LED light was on [46]. For the behavioral test on the agar dishes the blue light was $133 \mathrm{Mols}^{-1} \mathrm{~m}^{-2}$ per A $\left(28.9 \mathrm{Wmm}^{-2}\right)$. The adult flies, females and males, were anesthetized by exposing them to $\mathrm{CO}_{2}$ and placing them in a glass Petri dish or into a plastic vial for behavioral analysis during exposure to blue light. The flies were left for $10 \mathrm{~min}$ to recover. The activity was recorded on the camcorder mentioned above [47]. Low light conditions were used as best as possible. The adults were allowed to dark-adapt for 10 min prior to recording in IR light.

\subsection{Immunocytochemistry}

Preparations were dissected in normal HL3 saline and held in a stretch position while incubating to Bouin's fixative for $10 \mathrm{~min}$ and exchanged with fresh Bouin's fixative for another $10 \mathrm{~min}$. The preparations were placed in glass specimen vials and bathed three times with HL3 saline for 20 min each time on a shaker. For the fourth rinse in $5 \mathrm{~mL}$ of HL3 saline, 1 drop of Triton- $X$ was added and the preparations were placed back on a shaker. The preparations were bathed again in HL3 saline without Triton-X and $50 \mu \mathrm{L}$ of fetal calf serum was added to the $5 \mathrm{~mL}$ of HL3 saline for $20 \mathrm{~min}$ on a shaker. The bathing media was replaced with mouse anti-channelrhodopsin 2 (ChR2) monoclonal antibody (American Research Products, Inc; Waltham, MA 02452 USA; Catalog \#: 03-651180) at 1:250 in HL3 saline. The specimens were place at $4{ }^{\circ} \mathrm{C}$ on a shaker overnight. The preparations were rinses 3 times with HL3 saline for 20 min each time on a shaker at $4{ }^{\circ} \mathrm{C}$, then $50 \mu \mathrm{L}$ of fetal calf serum was added to the $5 \mathrm{~mL}$ of HL3 saline bathing the preparations and incubated for $20 \mathrm{~min}$ on a shaker. The media was exchanged for one with FITC conjugated goat anti-mouse IgG antibody at 1:120 in HL3 saline (Sigma; \#F-0257) and placed on a shaker overnight at $4{ }^{\circ} \mathrm{C}$. Preparations were mounted in Fluoromount-G (Southern Biotechnology Associates, Inc; Birmingham, AL 35209, USA) on glass slides and with a glass cover. The tissue was viewed and imaged on a Leica TCS SP8 DLS laser scanning confocal microscope with a $20 \times$ oil-immersion objective.

\subsection{Statistical Analysis}

A rank sum Wilcoxon test, non-parametric, was used to analyze changes in response to exposure to light. A significant difference is considered as $p<0.05$.

\subsection{Study Area}

The study was carried out in the Biology Department at the University of Kentucky in Lexington, KY, USA from May 2021 to July 2021. 
Supplementary Materials: The following are available online at https: / www.mdpi.com/article/ 10.3390/neuroglia2010007/s1. Movie S1: The activation of glia with channel rhodopsin in adult Drosophila (glia > Chr2 XXL). https: / youtu.be/eO0g5jTFwrM. Movie S2: Six adults of the parental line (UAS-ChR2XXL) and two adults expressing channel rhodopsin in glia (glia > Chr2 XXL) when exposed to blue light were all fed ATR for 2 days and maintained in the dark prior to viewing. Speed is in real time. https://youtu.be/NId7z7m-HrU. Movie S3: Adults of glia > Chr2 XXL in a glass Petri dish viewed from the top with IR light and a blue LED exposure for $30 \mathrm{~s}$. https:// youtu.be/Y2hVRb22-XU. Movie S4: To illustrate the rapid twitches while the adults were recovering from the paralysis a smaller dish was used with a higher magnification of glia > Chr2 XXL. https: / / youtu.be/bxZaHHZCeac. Movie S5: Having a dim white light background promoted the flies to move around on their own without having to mechanically shake the dish as for IR-only exposed adults. https: / /youtu.be/HZzSayAm6T8. Movie S6: The activation of glia with channel rhodopsin in larval Drosophila (glia > Chr2 XXL). https: / / youtu.be/6I7UDpmxi5Y. Movie S7: Larvae of the parental line (UAS-ChR2XXL) while exposed to blue light. https:/ / youtu.be/B-kSYQEXyvk.

Author Contributions: Data curation, R.L.C.; Formal analysis, C.P., S.M. and R.L.C.; Project administration, R.L.C.; Writing-original draft, C.P., S.M. and R.L.C. All authors contributed equally to this study. All authors have read and agreed to the published version of the manuscript.

Funding: Funding provided by University of Kentucky Neuroscience Research Priority Area to S.M. and Chellgren Endowed Funding to R.L.C. Stocks obtained from the Bloomington Drosophila Stock Center (NIH P40OD018537) were used in this study.

Institutional Review Board Statement: Not applicable.

Informed Consent Statement: Not applicable.

Data Availability Statement: All data are available in manuscript and available upon request.

Acknowledgments: We thank Nicholas McLetchie at the University of Kentucky for help using the LI-COR instrumentation for light measures. We thank James Begley at the University of Kentucky for help with confocal imaging and Doug Harrison (University of Kentucky) for comments on the Drosophila lines used.

Conflicts of Interest: The authors declare no conflict of interest.

\section{References}

1. Sancho, L.; Contreras, M.; Allen, N.J. Glia as sculptors of synaptic plasticity. Neurosci. Res. 2021, 167, 17-29. [CrossRef]

2. Einheber, S.; Bhat, M.; Salzer, J. Disrupted axo-glial junctions result in accumulation of abnormal mitochondria at nodes of Ranvier. Neuron Glia Biol. 2006, 2, 165-174. [CrossRef]

3. Baylor, D.A.; Nicholls, J.G. Changes in extracellular potassium concentration produced by neuronal activity in the central nervous system of the leech. J. Physiol. 1969, 203, 555-569. [CrossRef] [PubMed]

4. Jahromi, B.S.; Robitaille, R.; Charlton, M.P. Transmitter release increases intracellular calcium in perisynaptic Schwann cells in situ. Neuron 1992, 8, 1069-1077. [CrossRef]

5. Todd, K.J.; Darabid, H.; Robitaille, R. Perisynaptic glia discriminate patterns of motor nerve activity and influence plasticity at the neuromuscular junction. J. Neurosci. 2010, 30, 11870-11882. [CrossRef] [PubMed]

6. Robitaille, R. Purinergic receptors and their activation by endogenous purines at perisynaptic glial cells of the frog neuromuscular junction. J. Neurosci. 1995, 15, 7121-7131. [CrossRef] [PubMed]

7. Todd, K.J.; Robitaille, R. Purinergic modulation of synaptic signalling at the neuromuscular junction. Pflugers Arch. 2006, 452, 608-614. [CrossRef] [PubMed]

8. Auld, D.S.; Robitaille, R. Glial cells and neurotransmission: An inclusive view of synaptic function. Neuron 2003, 40, 389-400. [CrossRef]

9. Barik, A.; Li, L.; Sathyamurthy, A.; Xiong, W.C.; Mei, L. Schwann cells in neuromuscular junction formation and maintenance. J. Neurosci. 2016, 36, 9770-9781. [CrossRef]

10. Benraiss, A.; Wang, S.; Herrlinger, S.; Li, X.; Chandler-Militello, D.; Mauceri, J.; Burm, H.B.; Toner, M.; Osipovitch, M.; Xu, Q.J.; et al. Human glia can both induce and rescue aspects of disease phenotype in Huntington disease. Nat. Commun. 2016, 7, 11758. [CrossRef]

11. Onur, T.S.; Laitman, A.; Zhao, H.; Keyho, R.; Kim, H.; Wang, J.; Mair, M.; Wang, H.; Li, L.; Perez, A.; et al. Downregulation of glial genes involved in synaptic function mitigates Huntington's disease pathogenesis. eLife 2021, 10, e64564. [CrossRef] [PubMed]

12. Goldman, S.A.; Mariani, J.N.; Madsen, P.M. Glial progenitor cell-based repair of the dysmyelinated brain: Progression to the clinic. Semin. Cell Dev. Biol. 2021, 116, 62-70. [CrossRef] [PubMed] 
13. Kim, T.; Song, B.; Lee, I.S. Drosophila Glia: Models for Human Neurodevelopmental and Neurodegenerative Disorders. Int. J. Mol. Sci. 2020, 21, 4859. [CrossRef]

14. Ballinger-Boone, C.; Anyagaligbo, O.; Bernard, J.; Bierbower, S.M.; Dupont-Versteegden, E.E.; Ghoweri, A.; Greenhalgh, A.; Harrison, D.; Istas, O.; McNabb, M.; et al. The effects of bacterial endotoxin (LPS) on cardiac and synaptic function in various animal models: Larval Drosophila, crayfish, crab, and rodent. Int. J. Zool. Res. 2020, 16, 33-62. [CrossRef]

15. Dawydow, A.; Gueta, R.; Ljaschenko, D.; Ullrich, S.; Hermann, M.; Ehmann, N.; Gao, S.; Fiala, A.; Langenhan, T.; Nagel, G.; et al. Channelrhodopsin-2-XXL, a powerful optogenetic tool for low-light applications. Proc. Natl. Acad. Sci. USA 2014, 111, 13972-13977. [CrossRef]

16. Deisseroth, K. Optogenetics: 10 years of microbial opsins in neuroscience. Nat. Neurosci. 2015, 18, 1213-1225. [CrossRef]

17. Han, X.; Boyden, E.S. Multiple-color optical activation, silencing, and desynchronization of neural activity, with single-spike temporal resolution. PLoS ONE 2007, 2, e299. [CrossRef]

18. Sasaki, T.; Beppu, K.; Tanaka, K.F.; Fukazawa, Y.; Shigemoto, R.; Matsui, K. Application of an optogenetic byway for perturbing neuronal activity via glial photostimulation. Proc. Natl. Acad. Sci. USA 2012, 109, 20720-20725. [CrossRef]

19. Higgins, J.; Hermanns, C.; Malloy, C.; Cooper, R.L. Considerations in repetitive activation of light sensitive ion channels for long term studies: Channel rhodopsin in the Drosophila model. Neurosci. Res. 2017, 125, 1-10. [CrossRef]

20. Akasaka, T.; Ocorr, K. Drug discovery through functional screening in the Drosophila heart. Methods Mol. Biol. 2009, 577, 235-249. [CrossRef]

21. Bellen, H.J.; Tong, C.; Tsuda, H. 100 years of Drosophila research and its impact on vertebrate neuroscience: A history lesson for the future. Nat. Rev. Neurosci. 2010, 11, 514-522. [CrossRef] [PubMed]

22. Pandey, U.B.; Nichols, C.D. Human disease models in Drosophila melanogaster and the role of the fly in therapeutic drug discovery. Pharmacol. Rev. 2011, 63, 411-436. [CrossRef] [PubMed]

23. Stork, T.; Engelen, D.; Krudewig, A.; Silies, M.; Bainton, R.J.; Klämbt, C. Organization and function of the blood-brain barrier in Drosophila. J. Neurosci. 2008, 28, 587-597. [CrossRef]

24. MacNamee, S.E.; Liu, K.E.; Gerhard, S.; Tran, C.T.; Fetter, R.D.; Cardona, A.; Tolbert, L.P.; Oland, L.A. Astrocytic glutamate transport regulates a Drosophila CNS synapse that lacks astrocyte ensheathment. J. Comp. Neurol. 2016, 524, 1979-1998. [CrossRef]

25. Ito, K.; Urban, J.; Technau, G.M. Distribution, classification, and development of Drosophila glial cells in the late embryonic and early larval ventral nerve cord. Roux's Arch. Dev. Biol. 1995, 204, 284-307. [CrossRef] [PubMed]

26. Beckervordersandforth, R.M.; Rickert, C.; Altenhein, B.; Technau, G.M. Postembryonic development of the midline glia in the CNS of Drosophila: Proliferation, programmed cell death, and endocrine regulation. Mech. Dev. 2008, 125, 542-557. [CrossRef]

27. Awasaki, T.; Lai, S.-L.; Ito, K.; Lee, T. Organization and postembryonic development of glial cells in the adult central brain of Drosophila. J. Neurosci. 2008, 28, 13742-13753. [CrossRef]

28. Muthukumar, A.K.; Stork, T.; Freeman, M.R. Activity-dependent regulation of astrocyte GAT levels during synaptogenesis. Nat. Neurosci. 2014, 17, 1340-1350. [CrossRef]

29. Peco, E.; Davla, S.; Camp, D.; Stacey, S.M.; Landgraf, M.; van Meyel, D.J. Drosophila astrocytes cover specific territories of the CNS neuropil and are instructed to differentiate by Prospero, a key effector of Notch. Development 2016, 143, 1170-1181. [CrossRef]

30. Ng, F.S.; Tangredi, M.M.; Jackson, F.R. Glial cells physiologically modulate clock neurons and circadian behavior in a calciumdependent manner. Curr. Biol. 2011, 21, 625-634. [CrossRef] [PubMed]

31. Halassa, M.M.; Haydon, P.G. Integrated brain circuits: Astrocytic networks modulate neuronal activity and behavior. Annu. Rev. Physiol. 2010, 72, 335-355. [CrossRef]

32. Agulhon, C.; Fiacco, T.A.; McCarthy, K.D. Hippocampal short- and long-term plasticity are not modulated by astrocyte Ca ${ }^{2+}$ signaling. Science 2010, 327, 1250-1254. [CrossRef]

33. Liu, H.; Zhou, B.; Yan, W.; Lei, Z.; Zhao, X.; Zhang, K.; Guo, A. Astrocyte-like glial cells physiologically regulate olfactory processing through the modification of ORN PN synaptic strength in Drosophila. Eur. J. Neurosci. 2014, 40, 2744-2754. [CrossRef]

34. Xiang, Y.; Yuan, Q.; Vogt, N.; Looger, L.L.; Jan, L.Y.; Jan, Y.N. Light-avoidance-mediating photoreceptors tile the Drosophila larval body wall. Nature 2010, 468, 921-926. [CrossRef]

35. Arlow, R.L.; Foutz, T.J.; McIntyre, C.C. Theoretical principles underlying optical stimulation of myelinated axons expressing channelrhodopsin-2. Neuroscience 2013, 248, 541-551. [CrossRef]

36. Chamorro, E.; Bonnin-Arias, C.; Pérez-Carrasco, M.J.; Muñoz de Luna, J.; Vázquez, D.; Sánchez-Ramos, C. Effects of light-emitting diode radiations on human retinal pigment epithelial cells in vitro. Photochem. Photobiol. 2013, 89, 468-473. [CrossRef]

37. Lewis, E.B. A new standard food medium. Drosoph. Inf. Ser. 1960, 34, 117-118.

38. Brand, A.H.; Perrimon, N. Targeted gene expression as a means of altering cell fates and generating dominant phenotypes. Development 1993, 118, 401-415. [CrossRef] [PubMed]

39. Potter, S.; Sifers, J.; Yocom, E.; Blümich, S.L.E.; Potter, R.; Nadolski, J.; Harrison, D.A.; Cooper, R.L. Acute and chronic effects of inhibiting dTOR by rapamycin on development, behavior, and physiology in Drosophila. Biol. Open 2019, 8, bio046508. [CrossRef] [PubMed]

40. Govorunova, E.G.; Sineshchekov, O.A.; Janz, R.; Liu, X.; Spudich, J.L. Natural light-gated anion channels: A family of microbial rhodopsins for advanced optogenetics. Science 2015, 349, 647-650. [CrossRef] [PubMed]

41. Mauss, A.S.; Busch, C.; Borst, A. Optogenetic neuronal silencing in Drosophila during visual processing. Sci. Rep. $2017,7,13823$. [CrossRef] 
42. Zhao, S.; Cunha, C.; Zhang, F.; Liu, Q.; Gloss, B.; Deisseroth, K.; Augustine, G.J.; Feng, G. Improved expression of halorhodopsin for light-induced silencing of neuronal activity. Brain Cell Biol. 2008, 36, 141-154. [CrossRef]

43. Stewart, B.A.; Atwood, H.L.; Renger, J.J.; Wang, J.; Wu, C.F. Improved stability of Drosophila larval neuromuscular preparation in haemolymph-like physiological solutions. J. Comp. Physiol. A 1994, 175, 179-191. [CrossRef] [PubMed]

44. Muller, K.J.; Nicholls, J.G.; Stent, G.S. Neurobiology of the Leech; Cold Spring Harbor Laboratory: New York, NY, USA, $1981 ;$ p. 254.

45. DeCastro, C.; Titlow, J.; Majeed, Z.R.; Cooper, R.L. Analysis of various physiological salines for heart rate, CNS function, and synaptic transmission at neuromuscular junctions in Drosophila melanogaster larvae. J. Comp. Physiol. A 2014, $200,83-92$. [CrossRef] [PubMed]

46. DeCastro, N.; Cooper, R.L. Impedance measures and a mounting technique for drosophila: Larval movements, heart rate, imaging, and electrophysiology. Meth. Protoc. 2020, 3, 12. [CrossRef]

47. Majeed, Z.R.; Abdeljaber, E.; Soveland, R.; Cornwell, K.; Bankemper, A.; Koch, F.; Cooper, R.L. Modulatory action by the serotonergic system: Behavior and neurophysiology in Drosophila melanogaster. Neural Plast. 2016, 2016, 7291438. [CrossRef] [PubMed] 